S. YAMASHITA

KODAI MATH. J.

17 (1994), 142-162

\title{
BOU NDARY BEHAVIORS OF THE POINCARÉ DENSITY AND ITS DERIVATIVES NEAR A NONISOLATED BOUNDARY POINT
}

To Masatsugu Tsuji (1894-1960)

\author{
By SHINJI YAMASHITA
}

\begin{abstract}
Let $P_{\Omega}(z)|d z|$ be the Poincaré metric element with the constant Gauss curvature -4 of a hyperbolic domain $\Omega$ in the complex plane $\boldsymbol{C}$. We find some boundary properties of the Poincare density $P_{\Omega}$ and its complex partial derivatives $\left(P_{\Omega}\right)_{z},\left(P_{\Omega}\right)_{z z}$ and $\left(P_{\Omega}\right)_{z \bar{z}}$, in terms of the distance $\delta_{\Omega}(z)$ of $z \in \Omega$ and the boundary of $\Omega$ in $\boldsymbol{C}$. For the proof we make use of the sharp, lower estimates of $P \Omega_{(K)}$ of a domain $\Omega(K) \subset \boldsymbol{C}$ such that $K=\boldsymbol{C} \backslash \Omega(K)$ is a nondegenerate continuum. Several properties of the function $p(z, K), z \in \Omega(K)$, are proposed.
\end{abstract}

\section{Introduction}

A domain $\Omega$ in the complex plane $C=\{|z|<+\infty\}$ is called hyperbolic if its boundary $\partial \Omega$ in $C$ contains at least two points. Each hyperbolic domain $\Omega$ has the Poincare metric element $P_{\Omega}(z)|d z|, z \in \Omega$, that is, if $f$ is an analytic, universal-covering projection from the disk $D=\{|z|<1\}$ onto $\Omega, f \in \operatorname{Proj}(\Omega)$ in notation, then

$$
1 / P_{\Omega}(z)=\left(1-|w|^{2}\right)\left|f^{\prime}(w)\right|
$$

for the Poincare density $P_{\Omega}>0$ at $z=f(w), w \in D$. The choice of $f$ and $w$ is immaterial as far as $z=f(w)$ is satisfied.

It is familiar that $P_{\Omega}(z)$ tends to $+\infty$ as $z$ tends to each point $\zeta$ of $\partial \Omega[\mathrm{J}$, p. 116]. This also follows from a more precise property:

$$
\liminf _{z \rightarrow \zeta}\left[\delta_{\Omega}(z) \log \left(1 / \delta_{\Omega}(z)\right)\right] P_{\Omega}(z) \geqq 1 / 2,
$$

where $\delta_{\Omega}(z)$ is the distance of $z \in \Omega$ and $\partial \Omega$; a proof is contained in Section 8 for completeness. In general, $\delta_{\Omega}(z) P_{\Omega}(z) \leqq 1$ at each point $z \in \Omega$; see [Kr, p. 45] and $[Y 2$, p. 104, (IP)] for example. In the forthcoming paper [Y4] we shall

1991 Mathematics Subject Classification. 30C99.

Received May 12, 1993. 
mainly study behaviors of $P_{\Omega},\left(P_{\Omega}\right)_{z}=\overline{\left(\overline{P_{\Omega}}\right)_{\bar{z}}},\left(P_{\Omega}\right)_{z \bar{z}}=\left(\overline{\left.P_{\Omega}\right)_{\bar{z} \bar{z}}}\right.$ and $\left(P_{\Omega}\right)_{z \bar{z}}=4^{-1} \Delta P_{\Omega}$ in the vicinity of an isolated boundary point $b$ in terms of $\delta_{\Omega}(z)$ which is $|z-b|$ near $b$. Here, $\phi_{z}=\partial \phi / \partial z=(1 / 2)\{(\partial \phi / \partial x)-i(\partial \phi / \partial y)\}, \phi_{\bar{z}}=\overline{(\bar{\phi})_{z}}, \phi_{z z}=\partial^{2} \phi / \partial z^{2}, \phi_{z \bar{z}}=$ $\partial^{2} \phi / \partial z \partial \bar{z}$, etc., are complex partial derivatives with respect to $z=x+\imath y$ and $\bar{z}=$ $x-i y$. In the present paper we investigate behaviors of them near a general or a nonisolated boundary point. We begin with

THEOREM 1. Let $\Omega \subset C$ be a hyperbolic domain and let $\zeta \in \partial \Omega$. Suppose that there exists a connected component $K$ of $C \backslash \Omega$ which contains $\zeta$ and another point. Suppose further that there exists an open disk $U$ of center $\zeta$ such that $U \cap(\boldsymbol{C} \backslash \Omega)$ $=U \cap K$. Then,

$$
\liminf _{z \rightarrow \zeta} \delta_{\Omega}(z) P_{\Omega}(z) \geqq 1 / 4
$$

Note that the following fact

$$
\left(P_{\Omega}\right)_{z \bar{z}}=P_{\Omega^{-1}}\left|\left(P_{\Omega}\right)_{z}\right|^{2}+P_{\Omega^{3}}^{3}>0
$$

in $\Omega$ is derived from the Gauss curvature identity

$$
\Delta \log P_{\Omega}=4 P_{\Omega}^{2} \quad \text { in } \Omega .
$$

THEOREM 2. Let $\Omega \subset \boldsymbol{C}$ be a hyperbolic domain and set

$$
\alpha_{\Omega}(\zeta)=\liminf _{z \rightarrow \zeta} \delta_{\Omega}(z) P_{\Omega}(z)
$$

for each $\zeta \in \partial \Omega$. Then,

$$
\liminf _{z \rightarrow \zeta} \delta_{\Omega}(z)^{2}\left|\left(P_{\Omega}\right)_{z}(z)\right| \leqq A\left(\alpha_{\Omega}(\zeta)\right) \leqq 1 / 2,
$$

where $A(x)=2\left(x-x^{2}\right), 0 \leqq x \leqq 1$;

$$
\liminf _{z \rightarrow \zeta} \delta_{\Omega}(z)^{3}\left|\left(P_{\Omega}\right)_{z z}(z)\right| \leqq B\left(\alpha_{\Omega}(\zeta)\right) \leqq \beta,
$$

where $B(x)=5 x^{3}-16 x^{2}+11 x, 0 \leqq x \leqq 1$ and

$$
\begin{gathered}
\beta=(182 \sqrt{91}-272) / 675=2.169 \cdots ; \\
\liminf _{z \rightarrow \zeta} \delta_{\Omega}(z)^{3}\left(P_{\Omega}\right)_{z \bar{z}}(z) \leqq C\left(\alpha_{\Omega}(\zeta)\right) \leqq 1,
\end{gathered}
$$

where $C(x)=5 x^{3}-8 x^{2}+4 x, 0 \leqq x \leqq 1$.

For our proof of Theorem 1 we need a detailed study of $P_{\Omega}$ in case $C \backslash \Omega$ is a nondegenerate continuum, that is, a closed and connected set in $C$ containing at least two points. For this purpose we introduce a function $p(z, K)$ of $z \in \boldsymbol{C} \backslash K$, where $K$ is a bounded, nondegenerate continuum and $\boldsymbol{C} \backslash K$ is connected. The function $p$ itself has some properties which would be worth proposing, and will be given mainly in the long Section 6 and in Section 7 . Our investiga- 
tion of $p$ culminates in Theorem 4 .

The existence condition of a disk $U$ described in Theorem 1 cannot be dropped to obtain (1); see Remark 2 in Section 5 . The absolute constant $1 / 4$ in (1) is best possible. Actually let $\Omega_{0}$ be the complement of the nonpositive real axis $(-\infty, 0]$ with respect to $C$. Then for $r>0$ and for real $\alpha,|\alpha|<\pi$, we have the expression:

$$
\begin{aligned}
\delta_{\Omega_{0}}\left(r e^{\imath \alpha}\right) P_{\Omega_{0}}\left(r e^{\imath \alpha}\right) & =1 /\{4 \cos (\alpha / 2)\}, \quad \text { if } \quad|\alpha| \leqq \pi / 2 ; \\
& =(1 / 2) \sin (|\alpha| / 2), \quad \text { if } \quad \pi / 2<|\alpha|<\pi .
\end{aligned}
$$

In particular, $\delta_{\Omega_{0}}(x) P_{\Omega_{0}}(x)=1 / 4$ for $x>0$, so that the lower limit in (1) at $\zeta=0$ for the present $\Omega_{0}$ is just $1 / 4$.

If $C \backslash \Omega$ is unbounded and it consists of a finite number of nondegenerate continua, then (1) is valid at each $\zeta \in \partial \Omega$. In particular we know further that $\inf _{z \in \Omega} \delta_{\Omega}(z) P_{\Omega}(z)>0$ [M, Lemma 2], or $\Omega$ is of finite type [Y1, Y2].

Let $\Omega$ be a hyperbolic domain in $C$. In the course of the proof of Theorem 2 we actually have the following at each $z \in \Omega$ :

$$
\begin{aligned}
& \delta_{\Omega}(z)^{2}\left|\left(P_{\Omega}\right)_{z}(z)\right| \leqq 1 / 2 ; \\
& \delta_{\Omega}(z)^{3}\left|\left(P_{\Omega}\right)_{z z}(z)\right| \leqq \beta ; \\
& \delta_{\Omega}(z)^{3}\left(P_{\Omega}\right)_{z \bar{z}}(z) \leqq 1
\end{aligned}
$$

The constants in the right-hand sides may not be sharp, yet the powers $k=2,3$ of $\delta_{\Omega}(z)^{k}$ are sharp. Actually,

$$
\begin{aligned}
& \lim _{z \rightarrow \zeta} \delta_{D}(z)^{2}\left|\left(P_{D}\right)_{z}(z)\right|=1 / 4 ; \\
& \lim _{z \rightarrow \zeta} \delta_{D}(z)^{3}\left|\left(P_{D}\right)_{z z}(z)\right|=1 / 4 ; \\
& \lim _{z \rightarrow \zeta} \delta_{D}(z)^{3}\left(P_{D}\right)_{z \bar{z}}(z)=1 / 4 .
\end{aligned}
$$

\section{Simply or doubly connected domains}

Given a nondegenerate continuum $K$ in $C$ we set

$$
\delta_{K}(z)=\inf _{w \in K}|z-w| \text { and } \Delta_{K}(z)=\sup _{w \in K}|z-w|
$$

for $z \in C$. Then $0 \leqq \delta_{K} \leqq \Delta_{K} \leqq+\infty$. If $\delta_{K}(z)=\Delta_{K}(z)$ at $z \in C \backslash K$, then $K$ lies on $\left\{w ;|w-z|=\delta_{K}(z)\right\}$ and has the length $\delta_{K}(z) \Theta_{K}(z)$ with $0<\Theta_{K}(z) \leqq 2 \pi$.

By $\Omega(K)$ we always mean a domain in $C$ such that $C \backslash \Omega(K)=K$ is a nondegenerate continuum. Thus, $\Omega(K)$ is hyperbolic, and further, $\Omega(K)$ is simply connected (doubly connected, respectively) if and only if $K$ is unbounded (bounded, respectively). We have $\delta_{\Omega(K)}(z)=\delta_{K}(z)>0$ at each $z \in \Omega(K)$. 
THEOREM 3. For $\Omega(K)$ defined in the preceding paragraph we have the following propositions (I) and (II):

(I) If $K$ of $\Omega(K)$ is unbounded, then

$$
\delta_{K}(z) P_{\Omega(K)}(z) \geqq 1 / 4 \quad \text { for all } z \in \Omega(K) .
$$

(II) Suppose that $K$ of $\Omega(K)$ is bounded and let $z \in \Omega(K)$.

(II.1) If $\delta_{K}(z)<\Delta_{K}(z)$, then

$$
\delta_{K}(z) P_{\Omega(K)}(z) \geqq \frac{\delta_{K}(z)^{1 / 2} / \Delta_{K}(z)^{1 / 2}}{4 \operatorname{arctanh}\left(\delta_{K}(z)^{1 / 2} / \Delta_{K}(z)^{1 / 2}\right)},
$$

where $\operatorname{arctanh} x=(1 / 2) \log [(1+x) /(1-x)], 0 \leqq x<1$.

(II.2) If $\delta_{K}(z)=\Delta_{K}(z)$, then

$$
\delta_{K}(z) P_{\Omega(K)}(z)=\left[\cos ^{2}\left(\Theta_{K}(z) / 4\right)\right] /\left[2 \log \left\{\operatorname{cosec}\left(\Theta_{K}(z) / 4\right)\right\}\right] .
$$

Actually Proposition (I) is well known because $\Omega(K)$ is simply connected; see $[\mathrm{Kr}$, p. 45$]$ and $[\mathrm{Y} 2$, p. 104, (IIP)] for example. As we have seen in Section 1 , the equality in (2.1) holds at all points $x>0$ in $\Omega_{0}=\Omega((-\infty, 0])$. The "limiting" case where $\Delta_{K}(z)=+\infty$, that is, $K$ is unbounded, "in" (2.2) is (2.1). An example of a pair $z, K$ for which the equality in (2.2) holds will be proposed.

Fix $\delta>0$ and $z \in C$. Let $V(z, \delta)=\{w ;|w-z|<\delta\}$ and let $K$ be a closed arc on the circle $\partial V(z, \delta)$ with $\Theta_{K}(z)<2 \pi$. Then, it follows from (2.3) that

$$
P_{\Omega(K)}(z) \longrightarrow 1 / \delta=P_{V(z, \delta)}(z)
$$

as $\Theta_{K}(z) \rightarrow 2 \pi$. Namely, at the very moment when $K$ separates $z$ from $\infty$, we have a continuous "change" (2.4). On the other hand, $P_{\Omega(K)}(z) \rightarrow 0$ as $\Theta_{K}(z) \rightarrow 0$. Namely, at the very moment when $\Omega(K)$ becomes nonhyperbolic, we "lose" $P_{\Omega(K)}$.

\section{Lemmata}

Let $\mathscr{S}(p)$ for $0<p<1$ be the family of meromorphic and univalent functions $f$ in $D$ with their common pole at $p$ and $f(0)=f^{\prime}(0)-1=0$. A typical member of $\mathscr{S}(p)$ is

$$
k_{p}(z)=p z /((p-z)(1-p z))
$$

which maps, in particular, the punctured disk

$$
D(p)=D \backslash\{p\}
$$

onto the domain $\Omega(K(p))$, where

$$
K(p)=\left[-p /(1-p)^{2},-p /(1+p)^{2}\right]
$$

is the closed real interval. Another typical one is $k_{p}^{*}$ explained later in the proofs of Lemma 2 and Theorem 4 . We begin with 
LEMMA 1. For $f \in \mathscr{S}(p), 0<p<1$,

$$
(C \cup\{\infty\}) \backslash f(D) \subset\left\{z ; p /(1+p)^{2} \leqq|z| \leqq p /(1-p)^{2}\right\} .
$$

Both bounds $p /(1 \pm p)^{2}$ in (3.1) are attained by $k_{p}$. Lemma 1 is due to W. Fenchel, W. E. Kirwan and G. Schober; see $[F ; K S]$ and [Go, p. 249, Theorem 41].

Suppose that $K$ of $\Omega(K)$ is bounded. Then, for each $z \in \Omega(K)$ there exists a meromorphic function $g$ in $D$ which maps $D$ univalently onto $\Omega(K) \cup\{\infty\}$ with $g(0)=z, g(p)=\infty, 0<p<1$. Suppose that $g_{1}$ is another with $g_{1}(0)=z$, $g_{1}(q)=\infty, 0<q<1$. Applying the Schwarz lemma to $g^{-1} \circ g_{1}$ and $g_{1}{ }^{-1} \circ g$ one can easily observe that $p=q$ and hence $g=g_{1}$. Thus, $g$ and $p$ both are unique. We shall call $g$ canonical for $z$ and $K$ and write $p=p(z, K)$. We may regard $(g-z) / g^{\prime}(0) \in \mathscr{S}(p)$ for the canonical $g$ for $z$ and $K$. Here we consider a geometrical bound for $p(z, K)$ in (3.2) below.

LEMMA 2. At each point $z$ of $\Omega(K)$ with bounded $K$ we have

$$
p(z, K) \geqq \frac{1-\left(\delta_{K}(z) / \Delta_{K}(z)\right)^{1 / 2}}{1+\left(\delta_{K}(z) / \Delta_{K}(z)\right)^{1 / 2}} .
$$

In case $\delta_{K}(z)=\Delta_{K}(z)$ we have

$$
p(z, K)=\sin \left(\Theta_{K}(z) / 4\right) .
$$

Proof. Apply Lemma 1 to $(g-z) / g^{\prime}(0) \in \mathscr{S}(p)$ for the canonical $g$ for $z$ and $K$ with $p=p(z, K)$. Then,

$$
\begin{gathered}
\delta_{K}(z) /\left|g^{\prime}(0)\right| \geqq p /(1+p)^{2} ; \\
\Delta_{K}(z) /\left|g^{\prime}(0)\right| \leqq p /(1-p)^{2},
\end{gathered}
$$

so that

$$
\delta_{K}(z) / \Delta_{K}(z) \geqq(1-p)^{2} /(1+p)^{2}
$$

shows (3.2). To see the sharpness let a constant $p$ with $0<p<1$ be given. Then, $k_{p}$ is canonical for 0 and $K(p)$, and $\delta_{K}(0)=p /(1+p)^{2}$ and $\Delta_{K}(0)=p /(1-p)^{2}$. Now the equality in (3.2) holds for the pair $z=0$ and $K=K(p)$.

In case $\delta=\delta_{K}(z)=\Delta_{K}(z)$ we let $z+\delta e^{2 \alpha}$ and $z+\delta e^{i \beta}$ be the initial and terminal points of the arc $K$, so that $\beta-\alpha=\Theta_{K}(z)<2 \pi$. Set

$$
c=\tan \left(\Theta_{K}(z) / 4\right) \text { and } b=\left(\left(c^{2}+1\right)^{1 / 2}-1\right) / c .
$$

Let $\zeta=g(w)$ be the composed function of the following four:

$$
\begin{gathered}
w_{1}=(w-b) /(1-b w), \quad w \in D ; \\
w_{2}=(c / 2)\left(w_{1}-w_{1}^{-1}\right) ; \\
w_{3}=\left(1-w_{2}\right) /\left(1+w_{2}\right) ;
\end{gathered}
$$




$$
\zeta=z+\delta w_{3} e^{2(\alpha+\beta) / 2} .
$$

Then $g$ is canonical for $z$ and $K$ with $g(b)=z-\delta e^{\imath(\alpha+\beta) / 2}$ and $g(p)=\infty$, where

$$
p=p(z, K)=2 b /\left(b^{2}+1\right)=\sin \left(\Theta_{K}(z) / 4\right) .
$$

To be more explicit, we define for general $p, 0<p<1$, the function

$$
k_{p}^{*}(z)=p z(1-p z) /(p-z), \quad z \in D .
$$

Then $k_{p}^{*} \in \mathscr{S}(p)$, and, for the specified $p=\sin ((\beta-\alpha) / 4)$, we have the exact form of $g$ :

$$
g=z-\left(p^{-1} \delta e^{2(\alpha+\beta) / 2}\right) k_{p}^{*} \quad \text { in } \quad D .
$$

\section{Proof of Theorem 3}

If $K$ of $\Omega(K)$ is bounded, then

$$
\delta_{K}(z) P_{\Omega(K)}(z) \geqq(p-1) /(2(1+p) \log p)
$$

at each $z \in \Omega(K)$ with $p=p(z, K)$. For the proof we let $g$ be canonical for $z$ and $K$, and further, $f \in \operatorname{Proj}(D(p))$ with $f(0)=0$. Then $g \circ f \in \operatorname{Proj}(\Omega(K))$ with $z=g \circ f(0)$. Hence

$$
1 / P_{\Omega(K)}(z)=\left|g^{\prime}(0) f^{\prime}(0)\right|=\left|g^{\prime}(0)\right| / P_{D(p)}(0),
$$

so that

Since

$$
\delta_{K}(z) P_{\Omega(K)}(z)=P_{D(p)}(0) \delta_{K}(z) /\left|g^{\prime}(0)\right|
$$

$$
P_{D(p)}(0)=\left(p^{2}-1\right) /(2 p \log p),
$$

one obtains (4.1) with the aid of (3.4). By the way, (3.5) yields for $p=p(z, K)$ that

$$
\Delta_{K}(z) P_{\Omega(K)}(z) \leqq(1+p) /(2(p-1) \log p) .
$$

An exact form of $f \in \operatorname{Proj}(D(p))$ with $f(0)=0$ is, for example, $f(w)=$ $\left.\phi_{p}\left(w+w_{p}\right) /\left(1+\overline{w_{p}} w\right)\right)$, where

$$
\psi_{p}(w)=\left[p+\exp \left(\frac{w+1}{w-1}\right)\right] /\left[1+p \exp \left(\frac{w+1}{w-1}\right)\right], \quad w \in D
$$

with

$$
w_{p}=(\log p+\pi i+1) /(\log p+\pi i-1) .
$$

The function $\psi_{p}$ will be considered again.

Proof of (II.1). We now have (2.2) by (4.1) and (3.2) because the righthand side of (4.1) is an increasing function of $p, 0<p<1$.

The function $k_{p}$ is canonical for 0 and $K(p)$ with $k_{p}^{\prime}(0)=1$, so that (4.2) 
yields :

$$
1 / P_{\Omega(K(p))}(0)=1 / P_{D(p)}(0)=(2 p \log p) /\left(p^{2}-1\right) .
$$

It is not difficult to prove that the equality in (2.2) holds for $K=K(p)$ and $z=0$.

Proof of (II.2). Let $g$ be the function of (3.7) considered in the proof of (3.3). Again, $g \circ \psi_{p} \in \operatorname{Proj}(\Omega(K))$, where $p=\sin \left(\Theta_{K}(z) / 4\right)$ by (3.3) and

$$
\left|g^{\prime}(0)\right|=\delta_{K}(z) \operatorname{cosec}\left(\Theta_{K}(z) / 4\right) \text {. }
$$

Hence

$$
1 / P_{\Omega(K)}(z)=\left(1 / P_{D(p)}(0)\right)\left|g^{\prime}(0)\right|
$$

yields (2.3).

Remark. Suppose that $\Omega$ is hyperbolic, unbounded, and $\partial \Omega$ is bounded. A typical example of $\Omega$ is $\Omega(K)$ with bounded $K$. Fix $a \in \partial \Omega$. Then, 0 is an isolated boundary point of

$$
\Omega *=\{1 /(z-a) ; z \in \Omega\}
$$

and for $z \in \Omega$,

$$
\left(|z-a|^{-1} \log |z-a|\right) P_{\Omega *}(1 /(z-a))=(|z-a| \log |z-a|) P_{\Omega}(z) .
$$

Since the left-hand side of the above equality tends to $1 / 2$ as $|z-a| \rightarrow+\infty$ (see the end of Section 8), the right-hand side has the limit $1 / 2$ as $|z| \rightarrow+\infty$. Since $\delta_{\Omega}(z) /|z-a| \rightarrow 1$ as $|z| \rightarrow+\infty$ it follows that

$$
\lim _{|z| \rightarrow+\infty}\left(\delta_{\Omega}(z) \log \delta_{\Omega}(z)\right) P_{\Omega}(z)=1 / 2 .
$$

In particular,

$$
\lim _{|z| \rightarrow+\infty} \delta_{\Omega}(z) P_{\Omega}(z)=0 .
$$

We cannot drop the boundedness of $\partial \Omega$ to have (4.4). Actually, with the aid of (1.3) one observes that

$$
\left(\delta_{\Omega_{0}}(z) \log \delta_{\Omega_{0}}(z)\right) P_{\Omega_{0}}(z) \longrightarrow+\infty
$$

as $|z| \rightarrow+\infty$ along each half line in $\Omega_{0}$ emanating from the origin. Furthermore. (4.5) is false for $\Omega_{0}$.

\section{Proofs of Theorems 1 and 2}

Proof of (1). For $U$ we may further assume that

$$
U=\{z ;|z-\zeta|<3 \varepsilon\} \quad(\varepsilon>0)
$$

satisfies $(\boldsymbol{C} \backslash U) \cap K \neq \varnothing$. Then, for each $z$ of 
we have

$$
U(\zeta, \varepsilon)=\{z \in \Omega ;|z-\zeta|<\varepsilon\}
$$

$$
\delta_{\Omega}(z)=\delta_{K}(z) \leqq \varepsilon<2 \varepsilon \leqq \Delta_{K}(z) \leqq+\infty .
$$

Here we remember that if $\Omega_{1} \subset \Omega_{2}$ then $P_{\Omega_{1}} \geqq P_{\Omega_{2}}$ in $\Omega_{1}$; see [Gl, p. 337]. Thus, $P_{\Omega}(z) \geqq P_{\Omega(K)}(z)$ at each $z \in \Omega$.

If $K$ is unbounded, then it immediately follows from (2.1) that

$$
\delta_{\Omega}(z) P_{\Omega}(z) \geqq \delta_{K}(z) P_{\Omega(K)}(z) \geqq 1 / 4
$$

at all $z \in U(\zeta, \varepsilon)$. Hence (1).

Suppose next that $K$ is bounded. Since

$$
\delta_{K}(z) / \Delta_{K}(z) \leqq \delta_{K}(z) /(2 \varepsilon) \leqq|z-\zeta| /(2 \varepsilon) \longrightarrow 0
$$

as $z \rightarrow \zeta$ in $U(\zeta, \varepsilon)$, it follows from (2.2) that

$$
\liminf _{z \rightarrow \zeta} \delta_{\Omega}(z) P_{\Omega}(z) \geqq \liminf _{z \rightarrow \zeta} \delta_{\Omega}(z) P_{\Omega(K)}(z) \geqq 1 / 4 .
$$

This is (1).

Proof of (2). We remember that for generel $\Omega$,

$$
2+\left|\left(P_{\Omega^{-1}}\right)_{z}\right| \leqq 2 \delta_{\Omega}{ }^{-1} P_{\Omega}^{-1} ;
$$

see $[Y 2, p .116,(7.3)]$. It then follows that

$$
\delta_{\Omega}{ }^{2}\left|\left(P_{\Omega}\right)_{z}\right| \leqq 2\left(\delta_{\Omega} P_{\Omega}-\delta_{\Omega}^{2} P_{\Omega}^{2}\right)=A\left(\delta_{\Omega} P_{\Omega}\right)
$$

in $\Omega$. We now have (2) by $A(x) \leqq 1 / 2$.

Proof of (3). For $f \in \operatorname{Proj}(\Omega)$ with $z=f(w)$ we have

$$
\begin{aligned}
& P_{\Omega}(z)^{-1}\left|\left(P_{\Omega}^{-1}\right)_{z z}(z)\right| \\
& \quad=(1 / 2)\left(1-|w|^{2}\right)^{2}\left|f^{\prime \prime \prime}(w) / f^{\prime}(w)-(3 / 2)\left(f^{\prime \prime}(w) / f^{\prime}(w)\right)^{2}\right| \\
& \quad \leqq 3\left(\delta_{\Omega}(z)^{-2} P_{\Omega}(z)^{-2}-1\right) ;
\end{aligned}
$$

see $[Y 1$, p. $168,(3.3) ;$ Y2, p. $113,(6.2)]$. Hence in $\Omega$,

$$
\delta_{\Omega}{ }^{3}\left|\left(P_{\Omega}\right)_{z z}\right| \leqq 2\left|\delta_{\Omega}{ }^{2}\left(P_{\Omega}\right)_{z}\right|^{2} \delta_{\Omega^{-1}} P_{\Omega}{ }^{-1}+3 \delta_{\Omega} P_{\Omega}-3 \delta_{\Omega}{ }^{3} P_{\Omega}{ }^{3} .
$$

Combining this with (5.1) one observes that the right-hand side is not greater than $B\left(\delta_{\Omega} P_{\Omega}\right) \leqq \beta$. Hence (3).

Proof of (4). It follows from (1.2) that

$$
\delta_{\Omega}{ }^{3}\left(P_{\Omega}\right)_{z \bar{z}}=\left|\delta_{\Omega}^{2}\left(P_{\Omega}\right)_{z}\right|^{2} \delta_{\Omega^{-1}} P_{\Omega}^{-1}+\left(\delta_{\Omega} P_{\Omega}\right)^{3} .
$$

The right-hand side is not greater than $C\left(\delta_{\Omega} P_{\Omega}\right) \leqq 1$. Hence (4). 
Remark 1. The forthcoming property (6.12), together with (4.1), also proves (1) in case $K$ is bounded.

Remark 2. For $n \geqq 9$ we set $a_{n}=1-n^{-1}, r_{n}=2^{-n}$, and we denote the closed real intervals by $I_{n}=\left[a_{n}-r_{n}, a_{n}+r_{n}\right]$. Then

$$
\Omega=D \backslash\left(\bigcup_{n=9}^{\infty} I_{n}\right)
$$

is a hyperbolic domain and for $n \geqq 9$,

$$
A_{n} \equiv\left\{z ; r_{n}<\left|z-a_{n}\right|<a_{n+1}-a_{n}-r_{n+1}\right\} \subset \Omega
$$

with

$$
\bmod A_{n} \equiv(2 \pi)^{-1} \log \left(\left(a_{n+1}-a_{n}-r_{n+1}\right) / r_{n}\right) \longrightarrow+\infty
$$

as $n \rightarrow+\infty$. It then follows from [BP, p. 478 , Corollary 1] that

$$
\inf _{z \in \Omega} \delta_{\Omega}(z) P_{\Omega}(z)=0
$$

At each $\zeta \in \partial \Omega \backslash\{1\}$ we may apply Theorem 1 to have (1). Hence

$$
\liminf _{z \rightarrow 1} \delta_{\Omega}(z) P_{\Omega}(z)=0 .
$$

There exists no $U$ described in Theorem 1 for $1 \in \partial \Omega$, where $K$ is the circle $\partial D$. Here we further note that

$$
\begin{aligned}
& \liminf _{z \rightarrow 1}|z-1| P_{\Omega}(z) \geqq 1 /\left(2 c_{H}\right) ; \\
& c_{H}=\Gamma(1 / 4)^{4} /\left(4 \pi^{2}\right)=4.376 \cdots ;
\end{aligned}
$$

see [Y4, Example 1 in Section 3]. The inequality $\delta_{\Omega}(z)<|z-1|$ for each $z \in \Omega$ near 1 with some unknown factors might yield this delicate difference between (5.2) and (5.3).

\section{Further about $p(z, K)$}

The disk $D$ is a metric space with the distance (a bad terminology is the pseudodistance):

$$
d(\zeta, \eta)=|\zeta-\eta| /|1-\bar{\zeta} \eta|, \zeta, \eta \in D
$$

see [T, p. 511] for the proof of the triangle inequality. Each conformal mapping from $D$ onto $D$ preserves the distance $d$. Throughout in the present section we assume that $K$ of $\Omega(K)$ is bounded. We set

$$
d_{*}(z, w)=d(f(z), f(w))
$$

for $z, w \in \Omega *(K) \equiv \Omega(K) \cup\{\infty\}$, where $f$ is a conformal mapping from $\Omega *(K)$ 
onto $D$. The right-hand side of (6.1) is independent of the specified choice of $f$. In particular, for $g$ canonical for $z$ and $K$, we have

$$
p(z) \equiv p(z, K)=d(0, p(z))=d\left(g^{-1}(z), g^{-1}(\infty)\right)=d_{*}(z, \infty) .
$$

Since $\lim _{z \rightarrow \infty} p(z)=0$, we set $p(\infty)=0$. Hence, $p$ is a $C^{\infty}$ function in $\Omega(K)$; actually as will be observed, $p$ is real-analytic. Furthermore,

$$
|p(z)-p(w)| \leqq d_{*}(z, w) \quad \text { for } z, w \in \Omega *(K) .
$$

In other words, $p$ is a contraction from the metric space $\left(\Omega *(K), d_{*}\right)$ into the real interval $[0,1)$. The Poincaré distance of $z$ and $w$ in $\Omega *(K)$ is

$$
d_{\Omega *(K)}(z, w)=\operatorname{arctanh} d_{*}(z, w) .
$$

Again, $\left(\Omega *(K), d_{\Omega *(K)}\right)$ is a metric space. Hence, for $z, w \in \Omega^{*}(K)$,

$$
|\operatorname{arctanh} p(z)-\operatorname{arctanh} p(w)| \leqq d_{\Omega *(K)}(z, w) .
$$

Fix $z_{0} \in \Omega(K)$ and let $g_{0}$ be canonical for $z_{0}$ and $K$. Then,

$$
p(z)=d_{*}(z, \infty)=d\left(g_{0}^{-1}(z), p\left(z_{0}\right)\right), \quad z \in \Omega *(K) .
$$

Setting $h_{0}=g_{0}^{-1}$ in $\Omega^{*}(K)$ and then partially differentiating (6.4) with respect to $z$ in $\Omega(K)$, together with some calculations, we have

$$
2\left|p_{z}(z)\right| /\left(1-p(z)^{2}\right)=\left|h_{0}^{\prime}(z)\right| /\left(1-\left|h_{0}(z)\right|^{2}\right), \quad z \in \Omega(K) .
$$

Note that $|\operatorname{grad} \phi|=2\left|\phi_{z}\right|$ for a real function $\phi$. Letting $z \rightarrow z_{0}$ in (6.5) we then have

$$
\begin{gathered}
\left|\operatorname{grad} p\left(z_{0}\right)\right| /\left(1-p\left(z_{0}\right)^{2}\right)=1 /\left|g_{0}^{\prime}(0)\right|, \quad \text { or } \\
\quad \operatorname{grad} p(z)\left|/\left(1-p(z)^{2}\right)=1 /\right| g^{\prime}(0) \mid,
\end{gathered}
$$

where $g$ in (6.6), this time, is canonical for $z$ and $K$.

Proposition 1. For each $z \in \Omega(K)$ we have

$$
p(1-p) /\left((1+p) \delta_{K}\right) \leqq|\operatorname{grad} p| \leqq p(1+p) /\left((1-p) \Delta_{K}\right),
$$

where $p=p(z), \delta_{K}=\delta_{K}(z), \Delta_{K}=\Delta_{K}(z)$.

Proof. We have (6.7) from (6.6), (3.4) and (3.5). Consider

$$
k_{q}(w)=q w /((q-w)(1-q w))
$$

for $w \in D$ and $0<q<1$, which is canonical for 0 and the closed real interval $K(q)$. It then follows from (6.4) with $g_{0}=k_{q}$ and $z_{0}=0$ that

$$
p(z)=p(z, K(q))=d\left(k_{q}^{-1}(z), q\right), \quad z \in \Omega(K(q)) .
$$


Hence,

$$
p(0)=q \quad \text { and }|\operatorname{grad} p(0)|=1-q^{2} ;
$$

the latter follows from (6.6). It is now easy to prove that the equalities in (6.7) hold at $z=0$ for $K=K(q)$.

Q.E.D.

An application of (6.7) will be described in the remark after Corollary 1 to Theorem 4.

PROPOSITION 2. For each $z \in \Omega(K)$ we have

$$
P_{\Omega(K)}(z)=(1 / 2)|\operatorname{grad}\{\log (-\log p)\}|=|\operatorname{grad} p| /(-2 p \log p),
$$

where $p=p(z)$.

Proof. Remember (4.2): $P_{\Omega_{(K)}}(z)=P_{D(p)}(0) /\left|g^{\prime}(0)\right|$, where $g$ is canonical for $z$ and $K$. This, together with (6.6), yields (6.8).

Q.E.D.

PROPOSITION 3. Let $f: D \rightarrow \Omega(K)$ be analytic. Then

$$
\left(1-|z|^{2}\right)|(\partial / \partial z) p(f(z))| \leqq-p(f(z)) \log p(f(z))
$$

at each $z \in D$.

Note that $(\partial / \partial z) p(f(z))=p_{\zeta}(\zeta) f^{\prime}(z), \zeta=f(z)$.

Proof. We choose $F \in \operatorname{Proj}(\Omega(K))$ with $F(0)=f(z)$. Apply the Schwarz lemma: $\left|h^{\prime}(0)\right| \leqq 1$ to a branch $h(w)$, with $h(0)=0$, of the function $F^{-1} \circ f((w+$ $z) /(1+\bar{z} w))$ of $w \in D$. Then, since $1 /\left|F^{\prime}(0)\right|=P_{\Omega(K)}(f(z))$, it follows that

$$
\left(1-|z|^{2}\right)\left|f^{\prime}(z)\right| P_{\Omega(K)}(f(z)) \leqq 1,
$$

which, combined with (6.8), shows (6.9). The equality in (6.9) at $z$ (actually, then at all $z \in D$ ) holds if and only if $h(w) \equiv \varepsilon w, \varepsilon \in \partial D$, and hence, if and only if $f \in \operatorname{Proj}(\Omega(K))$.

Q.E.D.

It immediately follows from (6.9) that,

$$
2|(\partial / \partial z) \log (-\log p(f(z)))| \leqq 2 /\left(1-|z|^{2}\right), \quad z \in D,
$$

for analytic $f: D \rightarrow \Omega(K)$. Hence for $z, w \in D$,

$$
|\log (\{\log p(f(z))\} /\{\log p(f(w))\})| \leqq 2 \operatorname{arctanh} d(z, w) .
$$

Another consequence of $(6.8)$ is that

$$
|\log (\{\log p(z)\} /\{\log p(w)\})| \leqq 2 d_{\Omega(K)}(z, w)
$$

for $z, w \in \Omega(K)$, where 


$$
d_{\Omega(K)}(z, w)=\inf _{r} \int_{r} P_{\Omega(K)}(\zeta)|d \zeta|,
$$

$\gamma$ ranging over all recctifiable curves connecting $z$ and $w$ in $\Omega(K)$. We remember that $d_{\Omega(K)}$ is the Poincaré distance in $\Omega(K)$. There exists a not necessarily unique curve $\gamma_{0}=\gamma_{0}(z, w) \subset \Omega(K)$ connecting $z$ and $w$ with $z \neq w$ in $\Omega(K)$ such that

$$
d_{\Omega(K)}(z, w)=\int_{r_{0}} P_{\Omega(K)}(\zeta)|d \zeta| .
$$

Note that $\log (-\log p)$ is superharmonic in $\Omega(K)$. In fact, for $p=p(z), z \in \Omega(K)$,

$$
\Delta \log (-\log p)=-|\operatorname{grad} p|^{2} /(p \log p)^{2}=-4 P_{\Omega(K)}(z)^{2} .
$$

Since the derivative of $g_{0}^{-1}$ in (6.4) never vanishes in $\Omega(K)$, the function $\log |\operatorname{grad} p|$ is harmonic in $\Omega(K)$. Consider the metric $\lambda_{\Omega(K)}(z)|d z|$ with the density

$$
\lambda_{\Omega(K)}=|\operatorname{grad} p| /\left(1-p^{2}\right)=|\operatorname{grad} \operatorname{arctanh} p|, p=p(z),
$$

in $\Omega(K)$. In view of (6.5), $\lambda_{\Omega(K)}(z)|d z|$ is actually the restriction to $\Omega(K)$ of the Poincare metric element of $\Omega *(K)$. In particular, with the aid of (6.5) one observes that

$$
-\lambda_{\Omega(K)}(z)^{-2} \Delta \log \lambda_{\Omega(K)}(z) \equiv-4,
$$

or the Gauss curvature of $\lambda_{\Omega(K)}(z)|d z|$ at each $z \in \Omega(K)$ is constantly -4 . Remember that the Gauss curvature of $P_{\Omega(K)}(z)|d z|$ is also constantly -4 .

Proposition 4. For $z \neq w$ in $\Omega(K)$,

$$
|\operatorname{arctanh} p(z)-\operatorname{arctanh} p(w)|<d_{\Omega(K)}(z, w) .
$$

Proof. Since $\Omega(K) \subset \Omega *(K)$ we have $\lambda_{\Omega(K)}<P_{\Omega(K)}$ in $\Omega(K)$, so that (6.11) is immediate. However, we shall give a self-contained proof. First,

Hence,

$$
P_{\Omega(K)}(z) / \lambda_{\Omega(K)}(z)=P_{D(p(z))}(0)>1 \quad \text { in } \Omega(K) .
$$

$|\operatorname{arctanh} p(z)-\operatorname{arctanh} p(w)|$

$$
\leqq \int_{\gamma_{0}(z, w)} \lambda_{\Omega(K)}(\zeta)|d \zeta|<\int_{r_{0}(z, w)} P_{\Omega(K)}(\zeta)|d \zeta|=d_{\Omega(K)}(z, w) .
$$

Q.E.D.

Now, we have $\delta_{K}(z) / \Delta_{K}(z) \rightarrow 0$ as $z \rightarrow b \in \partial \Omega(K)$ in $\Omega(K)$. For, $\Delta_{K}(z)$ is bounded away from zero as $z \rightarrow b$. Hence (3.2) with $p<1$ yields that

$$
\lim _{z \rightarrow b} p(z)=1, \quad b \in \partial \Omega(K) .
$$

Suppose that there exists a rectifiable curve $r \subset \Omega(K)$ with a starting point 
$w \in \Omega(K)$ and an ending point $b \in \partial \Omega(K)$. Then,

$$
\int_{\gamma} P_{\Omega(K)}(\zeta)|d \zeta|=\int_{r} \lambda_{\Omega(K)}(\zeta)|d \zeta|=+\infty .
$$

For the proof we let $\gamma(z)$ be a subarc of $\gamma$ with the starting point $w$ and an ending point $z \in \gamma$. Then, the estimate:

$$
|\operatorname{arctanh} p(z)-\operatorname{arctanh} p(w)| \leqq \int_{r(z)} \lambda_{\Omega(K)}(\boldsymbol{\zeta})|d \zeta|,
$$

together with (6.12), proves (6.13). The situation is different for $\lambda_{\Omega(K)}$ and for a curve in $\Omega(K)$ ending at $\infty$. For each $z \in \Omega(K)$ we have an analytic curve $\Lambda(z) \subset \Omega(K)$ starting at $z \in \Omega(K)$ and ending at $\infty$ such that

$$
\int_{\Lambda(z)} \lambda_{\Omega(K)}(\zeta)|d \zeta|<+\infty
$$

For the proof we remember (6.5). Let $\Gamma(z)$ be the geodesic line segment between $h_{0}(z)$ and $p\left(z_{0}\right)$. Then for $\Lambda(z)=g(\Gamma(z))$ we have

$$
\int_{\Lambda(z)} \lambda_{\Omega(K)}(\zeta)|d \zeta|=\int_{\Gamma(z)}\left(1-|\eta|^{2}\right)^{-1}|d \eta|=d_{\Omega *(K)}(z, \infty)<+\infty .
$$

This is (6.14).

Let $\Lambda_{0}(z) \subset \Omega(K)$ be a locally rectifiable curve starting at $z \in \Omega(K)$ and ending at $\infty$. Then,

$$
\int_{\Lambda_{0}(z)} P_{\Omega(K)}(\zeta)|d \zeta|=+\infty
$$

For the proof we have only to let $w \rightarrow \infty$ along $\Lambda_{0}(z)$ in

$$
|\log (\{\log p(w)\} /\{\log p(z)\})| \leqq 2 \int_{\Lambda_{0}(z)} P_{\left.\Omega_{(K}\right)}(\zeta)|d \zeta| .
$$

Since $p(w) \rightarrow 0$ we have (6.15).

An important consequence of (6.4) is that $\log p$ is harmonic in $\Omega(K)$ and $\Delta p=4\left|p_{z}\right|^{2} / p$ in $\Omega(K)$; in fact, $-\log p(=+\infty$ at $\infty)$ in $\Omega *(K)$ is the Green function [N, p. 28 et seq., p. 123] of $\Omega *(K)$ with its pole at $\infty$. Another consequence of (6.4) is that the level set $\mathcal{L}(p, c) \equiv\{z \in \Omega(K) ; p(z)=c\} \quad(0<c<1)$ is the analytic Jordan curve which is the image by $g_{0}$, canonical for $z_{0}$ and $K$, of the Apollonius circle:

$$
\left\{w ; d\left(w, p\left(z_{0}\right)\right)=c\right\} .
$$

It follows from (6.12) that $\mathcal{L}(p, c)$ "separates" $\infty$ and $K$ : For each $c, 0<c<1$, $\{z \in \Omega(K) ; p(z) \leqq c\}$ is unbounded.

Set $\mathscr{D}(p, c)=\{z \in \Omega(K) ; p(z)<c\}$ for $0<c<1$, and let $h_{0}$ be the inverse of $g_{0}$ in (6.4). Then, $h_{0}(\mathscr{D}(p, c))=\left\{w ; 0<d\left(w, p\left(z_{0}\right)\right)<c\right\}$ has the non-Euclidean area $\pi c^{2} /\left(1-c^{2}\right)$. It then follows from (6.5) that 


$$
\iint_{\mathscr{D}(p, c)} \lambda_{\Omega(K)}(z)^{2} d x d y=\pi c^{2} /\left(1-c^{2}\right) .
$$

Since in $\mathscr{D}(p, c)$,

$$
P_{\Omega(K)}(z)>\left(\left(c^{2}-1\right) /(2 c \log c)\right) \lambda_{\Omega(K)}(z)
$$

it follows that

$$
\iint_{\mathscr{D}(p, c)} P_{\Omega(K)}(z)^{2} d x d y>\pi\left(1-c^{2}\right) /(2 \log c)^{2} .
$$

In particular,

$$
\lim _{c \rightarrow 1} \inf (1-c) \iint_{\mathscr{D}(p, c)} P_{\Omega(K)}(z)^{2} d x d y \geqq \pi / 2 ;
$$

note that $\mathscr{D}(p, c) \uparrow \Omega(K)$ as $c \uparrow 1$.

Similarly,

$$
\int_{\mathcal{L}(p, c)} \lambda_{\Omega(K)}(z)|d z|=2 \pi c /\left(1-c^{2}\right)
$$

and

$$
P_{\Omega(K)}(z)=\left(\left(c^{2}-1\right) /(2 c \log c)\right) \lambda_{\Omega(K)}(z)
$$

on $\mathcal{L}(p, c)$, so that

$$
\int_{\mathcal{L}(p, c)} P_{\Omega(K)}(z)|d z|=\pi /(-\log c),
$$

whence

$$
\lim _{c \rightarrow 1}(1-c) \int_{\mathcal{L}(p, c)} P_{\Omega(K)}(z)|d z|=\pi .
$$

This section now ends with a theorem and its corollaries.

THEOREM 4. Suppose that $K$ of $\Omega(K)$ is bounded and let $\mathcal{C}(K)$ be the capacity [N, p. 123] of K. Then,

$$
\mathcal{C}(K)^{-1} p^{2}\left(1-p^{2}\right) \leqq|\operatorname{grad} p| \leqq \mathcal{C}(K)^{-1} p^{2}\left(1-p^{2}\right)^{-1},
$$

where $p=p(z, K), z \in \Omega(K)$.

Proof. Let $g$ be canonical for $z$ and $K$. Since $-\log p(\zeta)=-\log d\left(g^{-1}(\zeta)\right.$, $p(z))$ is the Green function of $\Omega^{*}(K)$ with its pole at $\infty$, it follows on setting $\operatorname{Res}(g, p)=\lim _{w \rightarrow p}(w-p) g(w), p=p(z)$, that

$$
\mathscr{R}(K)=-\lim _{\zeta \rightarrow \infty}\left(\log d\left(g^{-1}(\zeta), p\right)+\log |\zeta|\right)=\log \left(\left(1-p^{2}\right) /|\operatorname{Res}(g, p)|\right)
$$

is the Robin constant [N, p. 123] of $\Omega *(K)$, so that $\mathcal{C}(K)=e^{-\mathcal{R}(K)}=|\operatorname{Res}(g, p)| /$ $\left(1-p^{2}\right)$ by definition; in particular, $|\operatorname{Res}(g, p(z))| /\left(1-p(z)^{2}\right)$ is independent of $z \in \Omega(K)$. Since $(g-z) / g^{\prime}(0) \in \mathscr{S}(p)$, it follows from [Ko, p. 278, (4.4)] (see also [Go, p. 263]) that 
Hence

$$
\left|g^{\prime}(0)\right| p^{2}\left(1-p^{2}\right) \leqq|\operatorname{Res}(g, p)| \leqq\left|g^{\prime}(0)\right| p^{2}\left(1-p^{2}\right)^{-1} \text {. }
$$

$$
\left|g^{\prime}(0)\right| p^{2} \leqq \mathcal{C}(K) \leqq\left|g^{\prime}(0)\right| p^{2}\left(1-p^{2}\right)^{-2},
$$

which, combined with (6.6), yields (6.16). Consider $k_{q}$ canonical for 0 and $K(q)$, $0<q<1$, for which we have $p(0)=q$ and $|\operatorname{grad} p(0)|=1-q^{2}$. Since $\operatorname{Res}\left(k_{q}, q\right)=$ $q^{2}\left(q^{2}-1\right)^{-1}$, it follows that $\mathcal{C}(K(q))=q^{2}\left(1-q^{2}\right)^{-2}$. This also follows from the fact that a rectilinear segment of length $a$ has the capacity $a / 4$; see [L, p. 172]. Hence the right equality in (6.16) holds at $z=0$ in $\Omega(K(q))$. We next consider the function $k_{q}^{*}$ of $\mathscr{S}(q), 0<q<1$; see (3.6). The function $k_{q}^{*}$ is then canonical for 0 and the circular arc

$$
K^{*}(q)=\left\{-q e^{i t} ;|t| \leqq 2 \Theta_{q}\right\},
$$

where $\Theta_{q}=\arcsin q \in(0, \pi / 2)$; in fact, $\Theta_{K^{*}(q)}(0)=4 \Theta_{q}$. Then, $p(0)=q$ and $|\operatorname{grad} p(0)|=1-q^{2}$ by $(6.6)$. Since $\operatorname{Res}\left(k_{q}^{*}, q\right)=q^{2}\left(q^{2}-1\right)$, it follows that $\mathcal{C}\left(K^{*}(q)\right)$ $=q^{2}$. Thus, the left-hand side equality in $(6.16)$ holds at $0 \in \Omega\left(K^{*}(q)\right)$. Q. E. D.

COROLLARY 1. Let $\mathcal{C}(K)$ be the capacity of $K$ and set $p(\zeta)=p(\zeta, K), \zeta \in \Omega(K)$. Then, $p+p^{-1}$ is Lipschit $z$ continuous:

$$
\left|\left(p(z)+p(z)^{-1}\right)-\left(p(w)+p(w)^{-1}\right)\right| \leqq \mathcal{C}(K)^{-1}|z-w|,
$$

$z, w \in \Omega(K)$.

Proof. For $\Phi=p+p^{-1}$ in $\Omega(K)$ the upper estimate of $|\operatorname{grad} p|$ in (6.16) yields that

$$
|\operatorname{grad} \Phi| \leqq \mathcal{C}(K)^{-1}
$$

For $z, w \in \Omega(K), z \neq w$, we consider the directed line from $w$ to $z$ :

$$
l(w, z)=\{\varphi(t) \equiv w+t(z-w) ; 0 \leqq t \leqq 1\} .
$$

Suppose first that $l(w, z) \cap K \neq \varnothing$ and then let

$$
l(1)=\left\{\varphi(t) ; 0 \leqq t<t_{1}\right\} \quad \text { and } \quad l(2)=\left\{\varphi(t) ; t_{2}<t \leqq 1\right\}, \quad\left(t_{1} \leqq t_{2}\right)
$$

be the connected components of $l(w, z) \cap \Omega(K)$ containing $w$ and $z$, respectively. Since $\Phi(\zeta) \rightarrow 2$ as $\zeta \in \Omega(K)$ tends to a point of $\partial \Omega(K)$ by (6.12), it follows that $\Phi(\varphi(t)) \rightarrow 2$ as $t \rightarrow t$, along $l(j), j=1,2$. Hence,

$$
\begin{gathered}
2-\Phi(w)=\int_{l(1)}\left(\Phi_{\xi}(\zeta) d \xi+\Phi_{\eta}(\zeta) d \eta\right), \\
\Phi(z)-2=\int_{l(2)}\left(\Phi_{\xi}(\boldsymbol{\zeta}) d \xi+\Phi_{\eta}(\boldsymbol{\zeta}) d \eta\right),
\end{gathered}
$$

where $\zeta=\xi+i \eta$. In view of $(6.18)$ one now obtains 


$$
\begin{aligned}
|\Phi(z)-\Phi(w)| & =\left|\int_{l(1) \cup l(2)}\left(\Phi_{\xi}(\zeta) d \xi+\Phi_{\eta}(\zeta) d \eta\right)\right| \\
& \leqq \int_{l(1) \cup l(2)}|\operatorname{grad} \Phi(\zeta)||d \zeta| \leqq C(K)^{-1} \int_{l(w, z)}|d \zeta|,
\end{aligned}
$$

whence (6.17). The case $l(w, z) \cap K=\varnothing$ is now obvious.

Q.E. D.

Let $w \rightarrow b \in \partial \Omega(K)$ in (6.17). It then follows from the resulting estimate that

$$
\begin{aligned}
& (1-p(z))^{2} / p(z) \leqq \mathcal{C}(K)^{-1} \delta_{K}(z) \equiv R(z), \quad \text { or } \\
& p(z) \geqq 2^{-1} R(z)+1-2^{-1}\left(R(z)^{2}+4 R(z)\right)^{1 / 2},
\end{aligned}
$$

$z \in \Omega(K)$; the right-hand side is positive and tends to 1 as $z$ tends to a point of $\partial(\Omega(K))$. The equality holds at $z=0$ for $K=K(q), 0<q<1$, because $\delta_{K(q)}(0)$ $=q /(1+q)^{2}$ and $\mathcal{C}(K(q))=q^{2} /\left(1-q^{2}\right)^{2}$.

Remark. It is not difficult to prove that

$$
\Delta(K) \equiv \inf _{z \in \Omega_{(K)}} \Delta_{K}(z)>0 .
$$

The upper estimate in (6.7) then yields that $|\operatorname{grad} \Psi| \leqq \Delta(K)^{-1}$ in $\Omega(K)$, where $\Psi=\log \left(p(1+p)^{-2}\right)$. We now have

$$
|\Psi(z)-\Psi(w)| \leqq \Delta(K)^{-1}|z-w|, \quad z, w \in \Omega(K),
$$

by the similar manner as in the paragraph just after the proof of Corollary 1. It is not difficult to have

whence

$$
p(z)^{-1}(1+p(z))^{2} \leqq 4 \exp \left(\Delta(K)^{-1} \delta_{K}(z)\right),
$$

$$
p(z) \geqq 2 Q(z)-1-2\left(Q(z)^{2}-Q(z)\right)^{1 / 2},
$$

where $Q(z)=\exp \left(\Delta(K)^{-1} \delta_{K}(z)\right), z \in \Omega(K)$; the right-hand side is positive and tends to 1 as $z$ tends to a point of $\partial \Omega(K)$.

COROLlaRY 2. At each $z \in \Omega(K)$ with $p=p(z, K)$, we have

$$
\mathcal{C}(K)^{-1} p\left(p^{2}-1\right)(2 \log p)^{-1} \leqq P_{\Omega(K)}(z) \leqq C(K)^{-1} p\left(2\left(p^{2}-1\right) \log p\right)^{-1} .
$$

Proof. This follows from (6.8) and (6.16). The right-hand side equality holds at 0 for $K=K(q)$ and the equality in the left holds at 0 for $K^{*}(q)$.

Q.E.D.

Remark. If $K$ of $\Omega(K)$ is further, convex, then the lower estimate in (6.16) can be replaced by

$$
\mathcal{C}(K)^{-1} p^{2}\left(1+p^{2}\right)^{-1} \leqq|\operatorname{grad} p|
$$


where $p=p(z, K), z \in \Omega(K)$. It is open whether or not the equality holds in (6.19). For the proof of (6.19), as in the proof of Theorem 4, we have only to make use of the estimate

$$
p^{2}\left(1+p^{2}\right)^{-1} \leqq|\operatorname{Res}(f, p)|,
$$

where $(C \cup\{\infty\}) \backslash f(D)$ for $f \in \mathscr{S}(p), 0<p<1$, is supposed to be convex (in the usual sense in $\left.\boldsymbol{R}^{2} \equiv \boldsymbol{C}\right)$ and again $\operatorname{Res}(f, p)=\lim _{z \rightarrow p}(z-p) f(z)$. Set $M=\operatorname{Res}(f, p)$ and consider the function:

$$
F(z)=M^{-1}\left(p^{2}-1\right) f((p-z) /(1-p z))=z^{-1}+a_{0}+a_{1} z+\cdots
$$

in $D$. Then $(C \cup\{\infty\}) \backslash F(D)$ is again convex. It then follows from [PP, p. 128, Corollary 5.1] (or [Go, p. 235, (45)] for $F(1 / z)$ ) that

$$
|M|^{-1}\left(p^{2}-1\right)^{2}|1-p z|^{-2}\left|f^{\prime}((p-z) /(1-p z))\right|=\left|F^{\prime}(z)\right| \leqq 1+|z|^{-2},
$$

$z \in D \backslash\{0\}$. Setting $z=p$ we now have $|M|^{-1} \leqq 1+p^{-2}$ or $(6.20)$.

\section{Once more on $p(z, K)$}

Again in this section we suppose that $K$ of $\Omega(K)$ is bounded. We prove the strict inequality

$$
\delta_{K}(z) P_{\Omega(K)}(z)<4 \sigma_{K}(z) /\left(\sigma_{K}(z)+1\right)^{2}(<1), \quad z \in \Omega(K),
$$

where

$$
\sigma_{K}(z)=\pi^{-1}\left[\log p+\left(\pi^{2}+(\log p)^{2}\right)^{1 / 2}\right] \quad(<1)
$$

with $p=p(z, K)$.

For the proof we let $g$ be canonical for $z$ and $K$, and we remember $\phi_{p}$ of (4.3) for $p=p(z, K)$. Then $f=g \circ \phi_{p} \in \operatorname{Proj}(\Omega(K))$ with $f\left(w_{p}\right)=z$. The supremum $\sigma_{K}(z)$ of $r, 0<r<1$, for which $f$ is univalent in

$$
\left\{w ;\left|w-w_{p}\right| /\left|1-\overline{w_{p}} w\right|<r\right\}
$$

is just that of $r, 0<r<1$, for which the function $e^{\zeta}$ is univalent in an Apollonius disk:

$$
\{\zeta ;|\zeta-\log p-\pi i| /|\zeta+\log p-\pi i|<r\}
$$

whose Euclidean diameter is

$$
(4 r \log p) /\left(r^{2}-1\right)
$$

Equating this with $2 \pi$ one has (7.2). The estimate (7.1) is just [Y2, p. 116, (7.4) for $\left.\rho_{\Omega(K)}(z)=\sigma_{K}(z)\right]$ which is strict in the present case.

It follows from [Y3, Theorem] that, for each $f$ analytic and univalent in $\Omega(K)$, the strict inequality holds : 


$$
\sigma_{K}(z) P_{\Omega(K)}(z)^{-1}\left|f^{\prime \prime}(z) / f^{\prime}(z)\right|<8, \quad z \in \Omega(K) .
$$

Combining this with (6.8) we have

$$
\tau_{K}(z)\left|f^{\prime \prime}(z) / f^{\prime}(z)\right|<8, \quad z \in \Omega(K),
$$

where

$$
\begin{aligned}
\tau_{K}(z) & =(-2 p \log p)\left[\log p+\left(\pi^{2}+(\log p)^{2}\right)^{1 / 2}\right] /|\pi \operatorname{grad} p| \\
& =(-p \log p)\left[\log p+\left(\pi^{2}+(\log p)^{2}\right)^{1 / 2}\right] /\left|\pi p_{z}\right|,
\end{aligned}
$$

with $p=p(z, K), z \in \Omega(K)$.

For $g_{0}$ in (6.4) we set

$$
f=\left(g_{0}^{-1}-p\left(z_{0}\right)\right) /\left(1-p\left(z_{0}\right) g_{0}{ }^{-1}\right)
$$

in $\Omega(K)$. Then $f$ is univalent and nonvanishing in $\Omega(K)$ with $p=|f|$. Consequently,

$$
f^{\prime} / f=2 p_{z} / p, \quad f^{\prime \prime} / f^{\prime}-f^{\prime} / f=p_{z z} / p_{z}-p_{z} / p,
$$

so that, we may consider (7.3) for

to have

$$
f^{\prime \prime} / f^{\prime}=p_{z z} / p_{z}+p_{z} / p
$$

$$
\tau_{K}(z)\left|p_{z z}(z, K) / p_{z}(z, K)+p_{z}(z, K) / p(z, K)\right|<8, \quad z \in \Omega(K) .
$$

Furthermore, it follows from [B, Corollary 3] (note: The last inequality in $[B$, Corollary 3$] \&[\mathrm{Hj}$, Theorem 1$] \Rightarrow[B G$, Theorem 1$] \Rightarrow$ The last inequality in $[\mathrm{B}$, Corollary 3$]$ ) that

$$
\left|f^{\prime \prime \prime}(z) / f^{\prime}(z)-(3 / 2)\left(f^{\prime \prime}(z) / f^{\prime}(z)\right)^{2}\right| \leqq 12 P_{\Omega(K)}(z)^{2}
$$

for all $z \in \Omega(K)$. A simple calculation, together with (6.8), now yields that, at each $z \in \Omega(K)$,

$$
\left|p_{z z z} / p_{z}-(3 / 2)\left\{\left(p_{z z} / p_{z}\right)^{2}+\left(p_{z} / p\right)^{2}\right\}\right| \leqq 12\left|p_{z}\right|^{2} /(p \log p)^{2}
$$

where $p=p(z, K)$.

We finish our study of $p(z)=p(z, K)$ with a proposition.

Proposition 5. Suppose that $K$ of $\Omega(K)$ is bounded. Then,

$$
\limsup _{z \rightarrow b}(1-p(z))^{2} P_{\Omega(K)}(z) \leqq 4^{-1} \mathcal{C}(K)^{-1}
$$

et each $b \in \partial \Omega(K)$;

$$
\begin{aligned}
& \lim _{z \rightarrow \infty} p(z)^{-1}(-\log p(z)) P_{\Omega(K)}(z)=2^{-1} \mathcal{C}(K)^{-1} ; \\
& \limsup _{z \rightarrow b}(1-p(z))^{4}\left|\left(P_{\Omega(K)}\right)_{z}(z)\right| \leqq 8^{-1} \mathcal{C}(K)^{-2}
\end{aligned}
$$


at each $b \in \partial \Omega(K)$;

$$
\limsup _{z \rightarrow \infty} p(z)^{-2}(-\log p(z))\left|\left(P_{\Omega(K)}\right)_{2}(z)\right| \leqq \pi^{-1} \mathcal{C}(K)^{-2} .
$$

Proof. Both (7.4) and (7.5) are consequences of Corollary 2 to Theorem 4. It follows from $[Y 2$, p. $116,(7.1)]$ that

$$
\left|\left(P_{\Omega(K)}\right)_{z}(z)\right| / P_{\Omega(K)}(z)^{2}<2 \sigma_{K}(z)^{-1},
$$

which, combined with (6.8), yields that

$$
\left|\left(P_{\Omega(K)}\right)_{z}(z)\right|<2^{-1}|\operatorname{grad} p|^{2}(p \log p)^{-2} \sigma_{K}(z)^{-1}, \quad p=p(z) .
$$

Thus, from the upper estimate in (6.16), the strict inequality follows:

$$
\left|\left(P_{\Omega(K)}\right)_{z}(z)\right|<2^{-1} \pi \mathcal{C}(K)^{-2} p^{2}\left(p^{2}-1\right)^{-2}(\log p)^{-2}\left[\log p+\left(\pi^{2}+(\log p)^{2}\right)^{1 / 2}\right]^{-1} .
$$

Both (7.6) and (7.7) now follow from this estimate.

Q.E.D.

\section{Behavior of $P_{\Omega}$ without any restriction on $\partial \Omega$}

In this section we prove (1.1) for a hyperbolic domain $\Omega$ in $C$. Let $a, b \in$ $\partial \Omega, a \neq b$, and let $w=\Phi(z)=(b-a) /(z-a)$. Then $\Phi(\Omega) \subset R \equiv C \backslash\{0,1\}$. It then follows from J. A. Hempel's result [Hm1, p. 443, (4.1)]:

$$
1 / P_{R}(w) \leqq 2|w|\left(|\log | w||+c_{H}\right), \quad w \in R,
$$

where $c_{H}$ is defined in (5.3), together with

$$
1 / P_{\Omega}(z)=|z-a|^{2} /\left(|b-a| P_{\Phi(\Omega)}(w)\right), \quad P_{\Phi(\Omega)}(w) \geqq P_{R}(w),
$$

that

$$
1 / P_{\Omega}(z) \leqq 2|z-a|\left(|\log (|b-a| /|z-a|)|+c_{H}\right)
$$

at each $z \in \Omega$.

For the proof of (1.1) we choose $b \in \partial \Omega \backslash\{\zeta\}$. Let

$$
\begin{aligned}
& V_{1}=\{z \in \Omega ;|z-\zeta|<2|b-\zeta| / 3\} ; \\
& V_{2}=\{z \in \Omega ;|z-\zeta|<|b-\zeta| / 3\} .
\end{aligned}
$$

Then for each $z \in V_{2}$ there exists $a=a(z) \in V_{1} \cap \partial \Omega$ (possibly $\zeta$ itself) such that

$$
\delta_{\Omega}(z)=|z-a| \leqq|z-\zeta|<|b-\zeta| / 3 .
$$

Since

$$
|b-\zeta| / 3 \leqq|b-a| \leqq 5|b-\zeta| / 3,
$$

it follows that 


$$
1 \leqq|b-a| / \delta_{\Omega}(z) \leqq 5|b-\zeta| /\left(3 \delta_{\Omega}(z)\right),
$$

which, combined with (8.1), shows that

$$
1 / P_{\Omega}(z) \leqq 2 \delta_{\Omega}(z)\left(\log \left[5|b-\zeta| /\left(3 \delta_{\Omega}(z)\right)\right]+c_{H}\right)
$$

for $z \in V_{2}$. Hence (1.1).

We have no reasonable estimate for the partial derivatives of $P_{\Omega}$.

As we have seen in [Y4], if $\zeta \in \partial \Omega$ is isolated, then

$$
\lim _{z \rightarrow \zeta}\left[\delta_{\Omega}(z) \log \left(1 / \delta_{\Omega}(z)\right)\right] P_{\Omega}(z)=1 / 2 .
$$

See [Ha, Section 9.4.3] and [Hm2, p. 104, Lemma 5.2]; the present author regrets overlooking the cited article $[\mathrm{Hm} 2]$ in [Y4].

\section{REFERENCES}

[B] J. Burbea, The Schwarzian derivative and the Poincaré metric, Pacific J. Math., 85 (1979), 345-354.

[BG] A.F. Beardon And F. W. Gehring, Schwarzian derivatives, the Poincaré metric and the kernel function, Comm. Math. Helv., 55 (1980), 50-64.

[BP] A.F. Beardon and C. Pommerenke, The Poincaré metric of plane domains, J. London Math. Soc. (2), 18 (1978), 475-483.

[F] W. Fenchel, Bemerkungen über die im Einheitskreis meromorphen schlichten Funktionen, Sitzsber. Preuss. Akad. Wiss. Phys.-Math. Kl., H22/23 (1931), 431-436.

[Gl] G. M. Goluzin, Geometric Theory of Functions of a Complex Variable, Amer. Math. Soc., Providence, 1969.

[Go] A. W. Goodman, Univalent Functions II, Mariner Publ., Tampa, 1983.

[Ha] W.K. HaYman, Subharmonic Functions II, Academic Press, London and 7 cities, 1989.

[Hj] D. A. Hejhal, Universal covering maps for variable regions, Math. Z., 137 (1974), $7-20$.

[Hm1] J.A. Hempel, The Poncare metric on the twice punctured plane and the theorems of Landau and Schottky, J. London Math. Soc. (2), 20 (1979), 435-445.

[Hm2] J. A. Hempel, On the uniformization of the $n$-punctured sphere, Bull. London Math. Soc., 20 (1988), 97-115.

[J] V. JøRGENSEN, On an inequality for the hyperbolic measure and its applications in the theory of functions, Math. Scand., 4 (1956), 113-124.

[Ko] Y. Komatu, Note on the theory of conformal representation by meromorphic functions I, II, Proc. Japan Acad., 21 (1945), 269-284.

[Kr] 1. KRA, Automorphic Forms and Kleinian Groups, Benjamin, Reading, 1972.

[KS] W.E. KirWAN AND G. SchOBER, Extremal problems for meromorphic univalent functions, J. Analyse Math., 30 (1976), 330-348.

[L] N.S. LANDKoF, Foundations of Modern Potential Theory, Springer, BerlinHeidelberg-New York, 1972.

[M] M. Masumoto, A distortion theorem for conformal mappings with an application to subharmonic functions, Hiroshima Math. J., 20 (1990), 341-350. 
[N] R. Nevanlinna, Eindeutige Analytische Funktionen, Springer, Berlin-GöttingenHeidelberg, 1953.

[PP] J. A. Pfaltzgraff and B. Pinchuk, A variational method for classes of meromorphic functions, J. Analyse Math., 24 (1971), 101-150.

[T] M. TsujI, Potential Theory in Modern Function Theory, Maruzen, Tokyo, 1959.

[Y1] S. Yamashita, Univalent analytic functions and the Poincaré metric, Kodai Math. J., 13 (1990), 164-175.

[Y2] S. Yamashita, The derivative of a holomorphic function and estimates of the Poincaré density, Kodai Math. J., 15 (1992), 102-121.

[Y3] S. Yamashita, La dérivée d'une fonction univalente dans un domaine hyperbolique, C. R. Acad. Sci. Paris., 314 (1992), 45-48.

[Y4] S. Yamashita, Sur allures de la densité de Poincaré et ses dérivées au voisinage d'un point frontière, Kodai Math. J., 16 (1993), 235-243.

Department of Mathematics

Tokyo Metropolitan University

Minami Ohsawa, Hachioji

TOKYO 192-03, JAPAN 